\title{
SÍNDROME METABÓLICO EN LOS TRABAJADORES DE LA UNIVERSIDAD PENÍNSULA DE SANTA ELENA - ECUADOR.
}

\author{
Autora: Dra. Bertha Maggi de Monserrate ${ }^{1}$ \\ Coautores: Hernán Moreano Andrade ${ }^{2}$ \\ Estudiantes del cuarto año de enfermería, módulo de Salud Pública, período lectivo $2011-2012^{3}$
}

1. Docente de la Carrera de Enfermería de Universidad Península de Santa Elena

2. Director del Instituto de Investigación Científica y Desarrollo Tecnológico de Universidad Estatal Península de Santa Elena.

3. Carrera de Enfermería de la Universidad Península de Santa Elena.

berthitamaggi2@hotmail.com

\begin{abstract}
Resumen
El Síndrome Metabólico es una patología que se caracteriza por presentar en un mismo individuo obesidad central, hipertrigliceridemia, colesterol HDL bajo, hiperglicemia e hipertensión arterial. Representa un factor de riesgo que predispone a Diabetes Mellitus 2 y enfermedades cardiovasculares. El objetivo general fue determinar la prevalencia de Síndrome Metabólico en los trabajadores de la Universidad Península de Santa Elena, con una muestra de 161 individuos (61,50\% hombres y 38,50\% mujeres). Basados en los criterios de la Federación Internacional de Diabetes, el 36.02\% de trabajadores, con una edad media de 43,81 $\pm 9,70$, presentó Síndrome Metabólico, mientras que de acuerdo a la American Panel Treatment III el 24, 22 \% de los trabajadores con una edad media de 43,79 $\pm 10,5$, presentó este síndrome. Basados en los criterios de la Federación Internacional de Diabetes la obesidad abdominal prevaleció en un 78, 26\% del total de los trabajadores con una media de 96,34 \pm 9,63 cm y de estos el 51,28\% trabajan administrativamente. En el diagnóstico del síndrome la Hipertrigliceridemia sobresalió con el 82,10\%.El Síndrome Metabólico prevaleció en los trabajadores que desempeñan un rol netamente administrativo.
\end{abstract}

Palabras claves: Síndrome Metabólico, trabajo administrativo.

\begin{abstract}
Metabolic syndrome is a condition that is characterized by the same individual central obesity, hypertriglyceridemia, low HDL cholesterol, hyperglycemia, and hypertension. It Represents a risk factor predisposing to diabetes mellitus 2 and cardiovascular diseases. The overall objective was to determine the prevalence of metabolic syndrome in workers at the University of Santa Elena Peninsula, with a sample of 161 individuals (61.50\% male and 38.50\% female). Based on the criteria of the International Diabetes Federation, $36.02 \%$ of the workers with an average age of $43.81 \pm 9.70$, presented the metabolic syndrome, while according to the American Treatment Panel III 24, 22\% of the workers with an average age of $43.79 \pm 10.5$, presented this syndrome. Based on the criteria of the International Diabetes Federation abdominal obesity prevailed by $78.26 \%$ of workers with a mean of $96.34 \pm 9.63 \mathrm{~cm}$ and of these, $51.28 \%$ worked administratively. In the diagnosis of the syndrome Hypertriglyceridemia excelled with $82.10 \%$. Metabolic syndrome prevails in workers performing purely administrative roles.
\end{abstract}

Key words: Metabolic Syndrome, Administrative Work. 


\section{Introducción}

Las Enfermedades crónicas no trasmisibles (ECNT) se encuentran seriamente vinculadas con el Síndrome Metabólico (SM), entidad reconocida desde los años 20 del siglo pasado, hasta nuestros días. Ha recibido diversas denominaciones a través del tiempo. En 1998 consultores de la Organización Mundial de la Salud (OMS) propusieron la denominación de SM [1]

Este síndrome es un estado patológico multifactorial, con una probabilidad muy alta de ser factor desencadenante de las enfermedades cardiovasculares (ECV) y de Diabetes Mellitus 2 (DM2), estas, componentes de las ECNT [2]

En el Ecuador seis de cada diez muertes corresponden a ECNT (Lizarzaburo, 2013) [3] y representaron el 63\% del número total de las muertes a nivel mundial. El 80\% de estas muertes se registraron en los países de ingresos bajos y medianos y más de nueve millones se produjeron en personas menores de 60 años, afectando por igual a hombres y mujeres [1]

El Sistema Integrado de Indicadores Sociales del Ecuador (SIISE) (2010) informó que en nuestro país el perfil de mortalidad por enfermedades infecciosas cambió por ECNT a partir de 1992, considerándose entre las primeras causas de mortalidad la DM2 y las Enfermedades Cerebrovasculares (ECV) [4].

El SM tiene factores genéticos que predisponen su desarrollo y se potencializan por factores adquiridos como el exceso de grasa corporal y la inactividad física [5].

A nivel mundial se ha constituido en un problema de salud pública. Los fallecimientos prematuros y las discapacidades resultantes ocasionan presupuestos sanitarios muy altos que podrían colapsar la economía tanto de los países desarrollados como los que se encuentran en vía de desarrollo [5]

Ford (2005) [6] describe que el SM podría ser el responsable de aproximadamente un $7 \%$ de la mortalidad por cualquier causa y hasta un $17 \%$ de las ECV.

De igual manera un informe del FraminghamHeartOffspringStudy (2005) mostró que la contribución de SM al riesgo cardiovascular y enfermedad coronaria era del
$34 \%$ y el $29 \%$ respectivamente, en los varones, y del $16 \%$ y el $8 \%$, respectivamente, en las mujeres [7].

Estas investigaciones mostraron un aumento del riesgo del $78 \%$ de accidentes cardiovasculares y muerte entre las personas con el SM. Los factores que más contribuyeron al síndrome fueron la Hipertensión con un 33\% y el nivel bajo de C HDL con un 25\% [5] [7].

La relación SM y DM2, en una revisión de estudios prospectivos reveló que el síndrome aumentó hasta el 52\% el riesgo de DM2. Ford (2005) [6].

La cohorte del estudio de Framingham descubrió que la presencia del SM al inicio del estudio era un potente indicador de DM2 en el futuro y que su contribución era del $62 \%$ en varones y $47 \%$ en mujeres (glucosa por encima de $100 \mathrm{mg} / \mathrm{dL}$ ), mientras que la combinación de hiperglicemia, obesidad abdominal y bajo nivel de C HDL asociaba a un riesgo 12 veces mayor de DM2 [8].

La importancia de combinaciones de factores de riesgo del SM que no incluyeron hiperglicemia transmitió un riesgo de unas cinco veces mayor de DM2, reflejando que la combinación de factores aumenta la sensibilidad a la insulina [7].

Epidemiológicamente este SM afecta entre un 20 a un $25 \%$ de la población mundial, y el porcentaje varia dependiendo los criterios diagnósticos que se utilicen [7]

En Estados Unidos se ha estimado la prevalencia de SM en un 22\%; 6,7\% en edades de 20 a 43 años y en un 43,5\% en personas mayores de 60 años, afectando por igual a ambos sexos, $23,4 \%$ para las mujeres y $24 \%$ para los hombres. En personas con familiares diabéticos la prevalencia aumenta a más del 80\% [9].

Harrison et al. (2007), determinaron que la prevalencia del SM en niños y adolescentes obesos quintuplica a la de los eutróficos (32,1\% vs $6,4 \%)$, la de los blancos triplica a la de los negros (7,1 vs $2,6 \%)$ y la de los insulinorresistentes duplica a la de los insulinosensibles (50\% vs $25 \%)$; por otra parte, esta patología aumentó en la última década tanto en población general (de 6,4\% a $10 \%)$ como en obesos $(28,7 \%$ a $32,1 \%)$ [10].

En Latinoamérica se han realizado estudios de $\mathrm{SM}$, demostrando que la prevalencia promedio es 
de 32,2\%; correspondiendo el 34\% a la población urbana y el 32.5\% a la rural (Consenso Latinoamericana de Diabetes, guía académica 2007 - 2010) [11], siendo México el país que tiene el mayor porcentaje de esta patología con un 50\% (Cuarta conferencia anual de Síndrome Metabólico (2012)) [12].

En el Ecuador se han realizado diversos estudios sobre SM entre ellos CARMELA que reveló una prevalencia promedio del 21\%, mientras que un estudio realizado en Pastaza en Indígenas Amazónicos la prevalencia fue del $18,5 \%$ [12].

En la Provincia de Santa Elena no se han encontrado estudios al respecto, sin embargo los datos epidemiológicos de SM ascienden a 384 casos desde el 2009 hasta el primer trimestre del 2013, de acuerdo al departamento de estadística de la Dirección provincial de Salud [13].

El SM esta estrechamente ligado a un estilo de vida poco saludable: alimentación tradicional rica en grasa saturada, exceso de carbohidratos y poca fibra, falta de ejercicio físico, estrés, y hábitos de alcoholismo, y tabaquismo, factores externos que desencadenan el factor genético para SM, propuesto por Poulsen(2001) [14].

Considerando que estos factores de riesgos son prevenibles, Burrowes (2006) [15] evidenció científicamente el beneficio de implementar una buena conducta nutricional y física para combatir el sobre peso y obesidad componente principal y desencadenante de la resistencia a la insulina (RI).

Consecuentemente la ingesta calórica deberá estar acorde a la composición corporal, sexo, edad, estado fisiológico o patológico, requerimientos del metabolismo basal y a la actividad que se desempeñe, Laguna (2007) [16]

En la Universidad Estatal Península de Santa Elena (UPSE) la tarea laboral se centra en tres actividades: administrativa, docencia y servicios varios. Bajo este contexto el grupo de docentes investigadores en ciencias de la salud estudiaron a los trabajadores de esta institución con el objetivo de determinar la prevalencia de SM y su relación con la actividad laboral.

\section{Materiales y métodos.}

La investigación correspondió a un estudio descriptivo transversal en una muestra tomada al azar de 161 trabajadores de la Universidad Estatal Península de Santa Elena, que asistieron al control periódico anual de salud 2011.

El instrumento para la recolección de datos fue un formulario elaborado para el efecto el cual contiene variables: demográficas, antecedentes patológicos personales (APP) y familiares (APF), metabólicas, antropométricas, hemodinámicas y de estilo de vida, adjuntando la hoja de consentimiento informado (Anexo 1).

En la variable demográfica se consideró la edad, sexo, estado civil, lugar de residencia y ocupación.

Los APP identificados fueron: DM2, Hipertensión Arterial (HTA), insuficiencia coronaria, accidente cerebro vascular, microalbuminuria, hiperuricemia, diabetes gestacional y ovario poliquístico.

Los APF identificados fueron: DM2, obesidad, accidente cerebrovascular, enfermedad coronaria, HTA y otros.

El perímetro abdominal se midió con cinta métrica plástica, graduada en $\mathrm{cm}$, en un punto equidistante entre el reborde costal y la espina iliaca antero - superior.

Se pesó a los trabajadores en kg empleando una Balanza, marca SECA, y la estatura en cm. mediante un Tallímetro de la misma marca. Las mediciones se realizaron en las primeras horas de la mañana.

Para la toma de presión arterial se utilizó un tensiómetro digital BHS (Riesterri - champion ${ }^{\circledR} \mathrm{N}$ ISO 9002), previo descanso del trabajador por 10 minutos en posición sentada.

La determinación de Glucosa, Colesterol total, HDL, LDL y triglicéridos fueron tomados previo ayuno de 8 a 12 horas. La medición se realizóen el analizador bioquímico BS - 380 MINDRAY del laboratorio clínico del Hospital del Instituto Ecuatoriano deSeguridad Social de Ancón, los reactivos fueron de la marca Human y cromatex respectivamente.

En el análisis de estilo de vida se consideró: nutrición, ejercicio y hábitos (tabaquismo y alcoholismo).

Para el diagnóstico de SM, se consideraron mínimo tres de los cinco criterios, propuestos por 
la Federación Internacional de Diabetes (IDF) y del Programa Nacional de Educación sobre el Colesterol y el Panel III del Tratamiento del Adulto

(NationalCholesterolEducationProgramAdultTreat ment Panel III) (Anexo 2).

\section{Resultados}

Se estudiaron 161 trabajadores de los cuales 62 fueron mujeres (38,50\%) y 99 hombres (61,50\%). Casados 100, solteros 45, unión libre 11 y divorciados 5. De ellos, 79 realizaban labor administrativa, 45 docencia, y 37 servicios varios. En cuanto al lugar de residencia, 58 vivían en Salinas, en La Libertad 55 y en Santa Elena 48.

Las edades fueron estratificadas de acuerdo a la tabla 1. Anexo 3. Respecto al estilo de vida: 12 trabajadores fuman (7,45\%); 31 ingieren alcohol $(19,25 \%)$ y 106 no realizan ejercicios $(65,84 \%)$.

Tabla 1. Edad de trabajadores por grupos etarios Grupos etarios $\quad \mathrm{N}^{\circ}$ de Trabajadores $\quad \%$

\begin{tabular}{|ccc|}
\hline $\mathbf{2 5} \mathbf{- 3 4}$ & 47 & 29,19 \\
\hline $\mathbf{3 5} \mathbf{- 4 4}$ & 58 & 36,02 \\
\hline $\mathbf{4 5}-\mathbf{5 4}$ & 36 & 22,36 \\
\hline $\mathbf{5 5} \mathbf{- 6 9}$ & 20 & 12,42 \\
\hline Total & 161 & 100 \\
\hline
\end{tabular}

\section{Comportamiento de los APP.}

La enfermedad más prevalente fue la HTA registrada en 14 trabajadores de los 53 quepresentaron algunapatología. Del 100\% de los trabajadores enfermos, solo el 28,30\% reciben tratamiento médico (Anexo 4).

\section{Comportamiento de los APF}

Los trabajadores que tuvieron familiares, de primera línea de consanguinidad, con DM2 e HTA, fueron 58 (36,02\%) y 27 tuvieron antecedentes de DM2 en familiares de segunda línea (16,77\%) (Anexo 5).

\section{Comportamiento de los indicadores diagnósticos.}

El Perímetro abdominal fue el indicador para valorar la obesidad central. De acuerdo al criterio de la IDF 126 trabajadores presentaron esta variable, (78,26\%), con una media de 96,34 \pm 9,63 cm. De los cuales 67 trabajaban administrativamente (53,17\%), 27 en docencia $(29,36 \%)$ y 22 en servicios varios (17,46\%). Mientras que cuando se aplicaron los criterios del ATP III, 57 trabajadores presentaron obesidad central, (35,40\%), la media fue de 102,61 \pm $9,21 \mathrm{~cm}$ y de ellos 29 realizaban tarea administrativa (51, 29\%), 12 docentes $\left(2^{\circ}, 52 \%\right)$ y 16 de servicios varios (28,20\%); notándose una diferencia del 42,86\% entre ambos criterios.

En la medición de los triglicéridos y glicemia,cuando se aplicaron ambos criterios, 83 trabajadorespresentaron Hipertrigliceridemia (51,60\%) siendo la media de $234 \pm 88,49$ e Hiperglicemia en 62 trabajadores(38,50\%), con una media de 118,82 \pm 38,92.

Cuando se midió el Colesterol HDL aplicando ambos criterios,55 trabajadores, (34,16\%), presentaron niveles bajos con una media de 38,52 $\pm 5,52$ y al valorar la HTA 18 presentaron HTAS, (11,18\%), con una media de $139 \pm 9,84$ e HTAD con una media de 91,05 $\pm 3,57$.

Se registró el IMC como un indicador anexo para la comparación del peso y la obesidad central determinando que 35 trabajadores con SM a pesar de tener su IMC normal, 1 presentó obesidad central cuando fue diagnosticado con los criterios de ATP III y 3 con el criterio de la IDF.

Las características clínicas de los indicadores de estudio se exponen en la tabla 2.

Tabla 2. Características clínicas de la muestra de acuerdo a los criterios de la IDF y ATP III.

\begin{tabular}{|c|c|c|c|c|c|}
\hline \multirow{2}{*}{ INDICADORES } & \multirow{2}{*}{$\begin{array}{l}\text { Total } \\
n=161\end{array}$} & \multicolumn{2}{|c|}{ IDF } & \multicolumn{2}{|c|}{ ATP III } \\
\hline & & $\begin{array}{l}58 \text { con } \\
\text { SM }\end{array}$ & $\begin{array}{l}111 \\
\mathrm{SM}\end{array}$ & $\begin{array}{l}39 \text { con } \\
\text { SM }\end{array}$ & $\begin{array}{l}122 \\
\mathrm{SM}\end{array}$ \\
\hline $\begin{array}{l}\text { Edad } \\
\text { Media }=\text { DS }\end{array}$ & $\begin{array}{l}41,48= \\
9,83\end{array}$ & $\begin{array}{l}43,18= \\
9,7\end{array}$ & $\begin{array}{l}40,17= \\
9,7\end{array}$ & $\begin{array}{l}43,79= \\
10,50\end{array}$ & $\begin{array}{l}40,14= \\
9,62\end{array}$ \\
\hline $\begin{array}{l}\text { IMC media } \pm \\
\text { DS }\end{array}$ & $\begin{array}{l}28,05 \pm \\
4,6\end{array}$ & $\begin{array}{l}29,53 \\
\pm \\
3,73 \\
\end{array}$ & $\begin{array}{l}27,22 \\
\pm 4.86\end{array}$ & $\begin{array}{l}30,59 \\
\pm 3,86\end{array}$ & $\begin{array}{l}27,10 \\
\pm 4,73\end{array}$ \\
\hline $\begin{array}{l}\text { PAB }(\mathrm{cm}) \text {, media } \\
=D S\end{array}$ & $\begin{array}{l}92,74= \\
11,38\end{array}$ & $\begin{array}{l}97,81= \\
8,99\end{array}$ & $\begin{array}{l}89,89= \\
11,63\end{array}$ & $\begin{array}{l}113,20 \\
=35,60\end{array}$ & $\begin{array}{l}89,42= \\
11,38\end{array}$ \\
\hline $\begin{array}{l}T \mathrm{~g} . \\
\text { media }=\mathrm{DS}\end{array}$ & $\begin{array}{l}170,37 \\
=93,49\end{array}$ & $\begin{array}{l}215,91 \\
=94,38\end{array}$ & $\begin{array}{l}154,08 \\
=82,83\end{array}$ & $\begin{array}{l}221,53 \\
=92,05\end{array}$ & $\begin{array}{l}152,50 \\
\pm 90,83\end{array}$ \\
\hline $\begin{array}{l}\text { Colesterol HDL } \\
\text { (mg/dL), media }= \\
\text { DS }\end{array}$ & $\begin{array}{l}49,27= \\
12,68\end{array}$ & $\begin{array}{l}43,13= \\
12,14\end{array}$ & $\begin{array}{l}52,72= \\
11,67\end{array}$ & $\begin{array}{l}43.02= \\
13.31\end{array}$ & $\begin{array}{l}52,29= \\
11,56\end{array}=$ \\
\hline $\begin{array}{l}\text { PAS }(\mathrm{mmHg}) \\
\text { media }=\mathrm{DS}\end{array}$ & $\begin{array}{l}117,66 \\
\pm 13,12\end{array}$ & $\begin{array}{l}121,34 \\
\pm 14,79\end{array}$ & $\begin{array}{c}115,01 \\
\pm 12,63\end{array}$ & $\begin{array}{l}125,10 \\
\pm 15,85\end{array}$ & $\begin{array}{l}115,48 \\
\pm 11,38\end{array}$ \\
\hline $\begin{array}{l}\mathrm{PAD} \quad(\mathrm{mmHg}) \\
\text { media }=\mathrm{DS}\end{array}$ & $\begin{array}{l}74,89= \\
9,65\end{array}$ & $\begin{array}{l}78,70= \\
10,75\end{array}$ & $\begin{array}{l}72,48= \\
8,76\end{array}$ & $\begin{array}{l}80,56= \\
09\end{array}$ & $\begin{array}{l}72,71= \\
8,35\end{array}$ \\
\hline
\end{tabular}




\section{Diagnóstico de Síndrome Metabólico}

El diagnóstico del SM se presenta en la Tabla 3.

Tabla 3. Diagnóstico de SM en los trabajadores de la UPSE.

\begin{tabular}{|c|c|c|c|c|c|c|c|c|c|c|}
\hline \multirow{3}{*}{$\begin{array}{l}\text { CRITERIO } \\
\text { DIAGNOSTICO }\end{array}$} & \multicolumn{10}{|c|}{ SÍNDROME METABÓUICO } \\
\hline & \multirow{2}{*}{$\%$} & \multirow{2}{*}{ Tresators } & \multirow{2}{*}{$\begin{array}{l}\text { EDAD } \\
\text { MEDIA }\end{array}$} & \multirow{2}{*}{$\begin{array}{c}\text { D. } \\
\text { STAND }\end{array}$} & \multicolumn{5}{|c|}{ PORCENTAUE } & \multirow[b]{2}{*}{ TOTAL } \\
\hline & & & & & $\mathrm{H}$ & $M$ & ADM & DOC & SV & \\
\hline IDF & 36,02 & 58 & 43,81 & $\pm 9,70$ & 69 & 31 & 55 & 21 & 24 & 100 \\
\hline ATP III & 24,22 & 39 & 43,79 & $\pm 10,50$ & 64 & 36 & 51 & 21 & 28 & 100 \\
\hline
\end{tabular}

Combinación de variables presentes en los Trabajadores con SM según los criterios IDF y NCEP -ATP III

La combinación de variables a considerar para el diagnóstico del SM se presenta en la Tabla 4.

Tabla 3. Combinación de variables a considerar para el diagnóstico del SM.

\begin{tabular}{|c|c|c|c|c|c|}
\hline \multirow{2}{*}{$\begin{array}{l}\text { COMBINACION DE } \\
\text { VARIABLES }\end{array}$} & \multirow{2}{*}{$\begin{array}{c}\text { No. } \\
\text { Variables }\end{array}$} & \multicolumn{2}{|c|}{ IDF } & \multicolumn{2}{|c|}{ ATP III } \\
\hline & & $\begin{array}{l}\mathrm{N}^{\circ} \\
\text { Trab. }\end{array}$ & $\%$ & $\begin{array}{l}\mathrm{N}^{\circ} \\
\text { Irab. }\end{array}$ & $\%$ \\
\hline $\begin{array}{l}\text { Obesidad, hipertigliceridemia, C } \\
\text { HDL bajo, hiperglicemia, HTA }\end{array}$ & 5 & 4 & 7 & 2 & 5 \\
\hline $\begin{array}{l}\text { Obesidad, hipertrigliceridemia, C } \\
\text { HDL bajo, hiperglicemia }\end{array}$ & 4 & 11 & 19 & 5 & 13 \\
\hline $\begin{array}{l}\text { Obesidad, hipertrigliceridemia, } \\
\text { HTA, hiperglicemia }\end{array}$ & 4 & 5 & 7 & 4 & 10 \\
\hline $\begin{array}{l}\text { Obesidad, C HDL bajo, HTA, } \\
\text { hiperglicemia }\end{array}$ & 4 & 1 & 2 & 1 & 3 \\
\hline $\begin{array}{l}\text { Obesidad, Hipertrigliceridemia, C } \\
\text { HDL bajo, HTA }\end{array}$ & 4 & 1 & 2 & 1 & 3 \\
\hline $\begin{array}{l}\text { Hipertrigliceridemia, C HDL } \\
\text { bajo, HTA, hiperglicemia }\end{array}$ & 4 & & & 2 & 5 \\
\hline $\begin{array}{l}\text { Obesidad, hipertrigliceridemia, } \\
\text { hiperglicemia. }\end{array}$ & 3 & 12 & 21 & 4 & 10 \\
\hline $\begin{array}{l}\text { Obesidad, hipertrigliceridemia, C } \\
\text { HDL }\end{array}$ & 3 & 11 & 19 & 5 & 13 \\
\hline $\begin{array}{l}\text { Obesidad, C HDL bajo, } \\
\text { Hiperglicemia }\end{array}$ & 3 & 10 & 17 & 5 & 13 \\
\hline $\begin{array}{l}\text { Obesidad, hipertrigliceridemia, } \\
\text { HTA }\end{array}$ & 3 & 2 & 3 & 2 & 5 \\
\hline Obesidad, HTA, Hiperglicemia & 3 & 1 & 2 & 1 & 3 \\
\hline $\begin{array}{l}\text { Hipertrigliceridemia, C HDL } \\
\text { bajo, hiperglicemia }\end{array}$ & 3 & & & 6 & 15 \\
\hline $\begin{array}{l}\text { Hipertrigliceridemia, HTA, } \\
\text { Hiperglicemia }\end{array}$ & 3 & & & 1 & 3 \\
\hline Total & & 58 & 100 & 39 & 100 \\
\hline
\end{tabular}

\section{Conclusiones.}

El análisis descriptivo determinó que el Síndrome Metabólico prevaleció en los trabajadores que desempeñan un rol netamente administrativo en la Universidad Península de Santa Elena.

\section{Recomendaciones.}

Formular modelos de estilo de vida saludable para los trabajadores, tanto para los que fueron diagnosticados con SM y los que no lo presentan, en base a tres componentes:

1.- Físico: Dosificación de ejercicios físicos terapéuticos, por grupos etarios y tipo de gestión y dosificación de ejercicios rutinarios para los trabajadores que no tienen SM.

2.- Nutricional: ingesta calórica acorde al tipo de gestión y su edad.

3.- Psicológico. Orientado a la reestructuración del pensamiento, mediante la teoría de valoración cognitiva.

Promocionar la importancia de los modelos de estilo de vida saludable para el empoderamiento de una cultura preventiva a través de los medios de difusión local.

\section{Referencias Bibliográficas}

1.Alberti KG, Zimmet PZ. 1998 Definition, diagnosis and classification of diabetes mellitus and its complications. Part 1: diagnosis and classification of diabetes mellitus provisional report of a WHO consultation. Diabet Med; 15: 539-53.

2. Kang YH, Min HG, Kim IJ, Kim YK, 2008. Son SM. Comparison of alanine aminotransferase, white blood cell count and uric acid in their association with metabolic syndrome: 
A study of corean adults. Endocr J;55 (6):1093102.

4. Sistema Integrado de Indicadores Sociales del Ecuador (SIISE) (2010).

5. http://www.elsevier.es/es/revistas/revis ta-espa\%C3\%B1ola-cardiologia-25/sindromemetabolico-concepto-fisiopatologia-13083442sindrome-metabolico-retos-esperanzas-2005.

6. Ford ES 2005. Risks for all-cause mortality, cardiovascular disease, and diabetes associated with the matabolicsíndrome: a sumary of the evidence. Diabetes Care; 28: 1769-78

7. Wilson PW, D'Agostino RB, Parise H, et al. 2005. Metabolic síndrome as a precursor of cardiovascular disease and type 2 diabetes Mellitus. Circulaton; 112:3066-72.

8. Síndrome metabólico - Diabetes y enfermedades - cardiovasculares seriamente vinculados. $\quad$ http//www.idf.org/diabetes vois/articles/

9. http://www.la granépoca.com/el-síndromemetabolico-ya-afecta-a-mas-del-20-de-lapoblación-mundial.

10. Harrison RI, Pratley RE, Bogardus C. '2007. The metabolic synoqudrome epidemiological approach. Am J Epidemiol; 151: 190 - 98.

11. Guía de Epidemiología, diagnóstico, control, prevención y tratamiento de Síndrome Metabólico programa académico 2007 - 2010, pág. 16.

12. Cuarta conferencia anual de Síndrome Metabólico

MD. F. 2012.

13. Departamento de estadística del la Dirección provincial de salud de Santa elena. 2013.

14. Poulsen P, Vaag A. 2001. Genetic versus environmental etiology of the metabolic síndrome among male and female twins. Diabetología; 44: 537-43.

15. Burrowes J.D. MetabolicSyndrome. NutritionToday 2006; 41(3): 131-137.
3. http://www.orasconhu.org/sites/default

/files/Plan\%20Estrategico\%20Quinquenal\%20201

2-2016.pdf

16. Laguna. 2007. Bioquímica de Laguna. 6ta edición. Editorial el Manuel Moderno. México D.F. 
ANEXOS 


\section{ANEXO 1}

\section{CARRERA DE ENFERMERÍA E INSTITUTO DE INVESTIGACIÓN CIENTÍFICA Y DESARROLLO TECNOLÓGICO (INCYT)}

\section{FORMULARIO PARA DETERMINAR SÍNDROME METABÓLICO EN LOS TRABAJADORES DE LA UNIVERSIDAD PENÍNSULA DE SANTA ELENA}

Elaborado por: Dra. Bertha Maggi ${ }^{1,2}$

1. Docente de la Carrera de Enfermería de la Universidad Península de Santa Elena

2. Instituto de Investigación Científica y Desarrollo Tecnológico (INCYT)

Fecha: La Libertad, --- de

de 2011

1. VARIABLES DEMOGRÁFICAS:

\begin{tabular}{|c|c|}
\hline Nombres completos & \\
\hline \multicolumn{2}{|l|}{ Edad (años) } \\
\hline Sexo & $\mathrm{H} \square$ \\
\hline Estado civil & S $\square$ C $\square$ $\square$ D $\square$ CS $\square$ \\
\hline \multicolumn{2}{|l|}{ Área de trabajo } \\
\hline \multicolumn{2}{|l|}{ Ocupación } \\
\hline Lugar de residencia & La Libertad $\square$ \\
\hline \multirow[t]{2}{*}{ Dirección } & Barrio o Ciudadela: \\
\hline & Calle \\
\hline Teléfonos & Fijo: \\
\hline Correo electrónico & \\
\hline
\end{tabular}

2. ANTECEDENTES PATOLÓGICOS PERSONALES

\begin{tabular}{|l|l|l|l|l|}
\hline PATOLOGÍAS & SI & NO & C. TRATAMIENTO & S. TRATAMIENTO \\
\hline Diabetes Mellitus & & & & \\
\hline Hipertensión Arterial & & & & \\
\hline Insuficiencia coronaria & & & & \\
\hline $\begin{array}{l}\text { Accidente vascular } \\
\text { cerebrovascular }\end{array}$ & & & & \\
\hline $\begin{array}{l}\text { Enfermedad } \\
\text { periférica }\end{array}$ & & & \\
\hline Microalbuminuria & & & & \\
\hline $\begin{array}{l}\text { Hiperuricemia (ácido } \\
\text { úrico elevado) }\end{array}$ & & & & \\
\hline Diabetes gestacional & & & & \\
\hline Ovario poliquístico & & & & \\
\hline
\end{tabular}

\section{ANTECEDENTES PATOLÓGICOS FAMILIARES}




\begin{tabular}{|l|l|l|l|l|l|l|}
\hline PATOLOGÍAS & Padre & Madre & Abuelos & Hermanos & Tíos & Primos \\
\hline Diabetes Mellitus & & & & & & \\
\hline Obesidad & & & & & & \\
\hline Accidente cerebrovascular & & & & & & \\
\hline Enfermedad coronaria & & & & & & \\
\hline Hipertensión arterial & & & & & & \\
\hline Otros & & & & & & \\
\hline
\end{tabular}

4. VARIABLES METABÓLICAS.

\begin{tabular}{|l|c|c|}
\hline EXAMEN & $\mathrm{Mg} / \mathrm{dl}$ & $\mathrm{mmol} / \mathrm{L}$ \\
\hline Glicemia en ayunas. & & \\
\hline
\end{tabular}

5. VARIABLES LIPÍDICAS

\begin{tabular}{|l|l|l|}
\hline EXAMEN & $\mathrm{Mg} / \mathrm{dl}$ & $\mathrm{mmol} / \mathrm{L}$ \\
\hline Colesterol total & & \\
\hline C. HDL & & \\
\hline C. LDL & & \\
\hline Triglicéridos & & \\
\hline
\end{tabular}

6. VARIABLES FÍSICAS

\begin{tabular}{|l|l|l|l|l|}
\hline $\begin{array}{l}\text { MEDIDAS } \\
\text { ANTROPOMÉTRICAS }\end{array}$ & $\mathrm{Kg}$ & $\mathrm{E} / \mathrm{cm}$ & $\mathrm{Kg} / \mathrm{m} 2$ & $\mathrm{PA} / \mathrm{cm}$ \\
\hline Peso & & & & \\
\hline Estatura & & & & \\
\hline IMC & & & & \\
\hline $\begin{array}{l}\text { Perímetro } \\
\text { abdominal }\end{array}$ & & & & \\
\hline
\end{tabular}

7. VARIABLES HEMODINÁMICAS

\begin{tabular}{|l|l|}
\hline PRESION ARTERIAL & $\mathrm{mmHg}$ \\
\hline Presión arterial sistólica & \\
\hline Presión arterial Diastólica & \\
\hline
\end{tabular}

8. VARIABLES DE ESTILO DE VIDA

\begin{tabular}{|l|l|l|}
\hline HÁBITOS & SI & NO \\
\hline Tabaquismo & & \\
\hline Alcoholismo & & \\
\hline Actividad física & & \\
\hline
\end{tabular}




\title{
CARRERA DE ENFERMERÍA E INSTITUTO DE INVESTIGACIÓN CIENTÍFICA Y DESARROLLO TACNOLÓGICO (INCYT)
}

\author{
HOJA DE CONSENTIMIENTO INFORMADO
}

Etapa1 de investigación.

“Determinación de Síndrome Metabólico en los Trabajadores de la Universidad Estatal Península de Santa Elena"

\section{INFORMACIÓN GENERAL}

Señor trabajador(a), lo(a) invitamos a participar en la determinación del síNDROME METABÓLICO. La intención es detectar la presencia deobesidad central, presión arterial elevada, aumento de azúcar y triglicéridos y disminución de Colesterol HDL en sangre, entre otros factores, mediante el examen físico y de laboratorio. Esta investigación está dirigida por la doctora Bertha Maggi, investigadora del INCYT en cooperación con los estudiantes de enfermería, los médicos del Departamento de Bienestar Universitario y el Hospital del IESS en Ancón.

Los beneficios de este estudio son diagnóstico, tratamiento y seguimiento del SíNDROME METABÓLICO, para evitar que usted sufra infarto del corazón o del cerebro o se convierta en un Diabético Mellitus 2 a corto plazo. Los trabajadores que ya padecen de Diabetes Mellitus 2 e Hipertensión Arterial, tendrán atención y prevención secundaria y terciaria de su enfermedad.

Los datos obtenidos a través de esta investigación serán utilizados para fines investigativos y se guardaran absoluta confidencialidad.

Su firma en este documento significa que ha decidido participar después de haber leído y discutido la información presentada en esta hoja de consentimiento.

Nombre del participante

Firma

Fecha

Dra. Bertha Maggi, M.Sc.

Directora de Investigación

Firma

Fecha 
Anexo 2. Criterios para el diagnóstico de Síndrome Metabólico

\begin{tabular}{|c|c|c|}
\hline Factor de Riesgo & IDF & ATP\|II \\
\hline ObesidadCentral & $\begin{array}{c}C A \geq 90 \mathrm{~cm} \\
\text { en hombres } y \geq 80 \mathrm{~cm} \text { en mujeres }{ }^{*}\end{array}$ & $\begin{array}{c}C A \geq 102 \mathrm{~cm} \\
\text { en hombres } y \geq 88 \mathrm{~cm} \text { en mujeres. }\end{array}$ \\
\hline Triacilglicéridos elevados & Igual o mayor a $150 \mathrm{mg} / \mathrm{dL}$ & Igual o mayor a $150 \mathrm{mg} / \mathrm{dL}$ \\
\hline ColesterolHDL Bajo & $\begin{array}{c}<40 \mathrm{mg} / \mathrm{dL} \text { en hombresy }<50 \mathrm{mg} / \mathrm{dL} \\
\text { en mujeres }\end{array}$ & $\begin{array}{c}<40 \mathrm{mg} / \mathrm{dL} \text { en hombres } \mathrm{c}<50 \mathrm{mg} / \mathrm{dL} \text { en } \\
\text { mujeres }\end{array}$ \\
\hline Hipertensiónarterial & Igual o mayor a $130 / 85 \mathrm{mmHg}$ & Igual o mayor a $130 / 85 \mathrm{mmHg}$ \\
\hline Glicemia alterada en ayunas & $\begin{array}{l}\text { Igual o mayor a 100mg/dL } 0 \\
\text { diagnóstico previo de DM. }\end{array}$ & Igual o mayor a $100 \mathrm{mg} / \mathrm{dL}$ \\
\hline
\end{tabular}

*Criterio obligatoriopara el diagnóstico. Datos obtenidos de las recomendaciones de la IDF para nativos de América centraly del Surhasta que existan más datos disponibles. 
ANEXO 3. COMPORTAMIENTO GENERAL DE LA MUESTRA POR EDAD SEXO, ESTADO CIVIL, OCUPACION Y LUGAR DE RESIDENCIA.

\begin{tabular}{|c|c|c|c|c|c|c|c|c|c|c|c|c|c|c|c|c|c|c|c|c|c|c|c|c|c|}
\hline \multirow{2}{*}{$\begin{array}{l}\text { GRUPOS } \\
\text { ETARIOS }\end{array}$} & \multirow{2}{*}{$\begin{array}{c}\mathbf{N}^{\circ} \mathrm{DE} \\
\text { TRABAJADORES }\end{array}$} & \multicolumn{4}{|c|}{ SEXO } & \multicolumn{8}{|c|}{ ESTADO CIVIL } & \multicolumn{6}{|c|}{ OCUPACIÓN } & \multicolumn{6}{|c|}{ LUGAR DE RESIDENCIA } \\
\hline & & H & $\%$ & $\mathbf{M}$ & $\%$ & $\mathbf{S}$ & $\%$ & C & $\%$ & UL & $\%$ & D & $\%$ & AD & $\%$ & DC & $\%$ & sv & $\%$ & SL & $\%$ & LL & $\%$ & SE & $\%$ \\
\hline $25-34$ & 47 & 26 & 55 & 21 & 45 & 17 & 36 & 22 & 47 & 4 & 8,5 & 4 & 8,5 & 26 & 55 & 8 & 17 & 13 & 28 & 16 & 34 & 19 & 40 & 12 & 26 \\
\hline $35-44$ & 58 & 32 & 55 & 26 & 45 & 15 & 26 & 38 & 66 & 5 & 8,6 & 0 & 0 & 26 & 45 & 21 & 36 & 11 & 19 & 20 & 34 & 18 & 31 & 20 & 34 \\
\hline $45-54$ & 36 & 25 & 69 & 11 & 31 & 10 & 28 & 24 & 67 & 1 & 2,8 & 1 & 2,8 & 16 & 44 & 11 & 31 & 9 & 25 & 14 & 39 & 11 & 31 & 11 & 31 \\
\hline $55-69$ & 20 & 16 & 80 & 4 & 20 & 3 & 15 & 16 & 80 & 1 & 5 & 0 & 0 & 11 & 55 & 5 & 25 & 4 & 20 & 8 & 40 & 7 & 35 & 5 & 25 \\
\hline TOTAL & 161 & 99 & 61 & 62 & 39 & 45 & 28 & 100 & 62 & 11 & 6,8 & 5 & 3,1 & 79 & 49 & 45 & 28 & 37 & 23 & 58 & 36 & 55 & 34 & 48 & 30 \\
\hline
\end{tabular}




\section{ANEXO 4.}

Tabla 2. Antecedentes Patológicos Personales \& Tratamiento Médico.

$\begin{array}{lllll}\text { Enferm. } & \text { Presente } & \% & \text { Con Trat. } & \% \\ \text { DM2 } & 5 & 9,43 & 3 & 20 \\ \text { HTA } & 14 & 26,42 & 6 & 40 \\ \text { IC } & 3 & 5,66 & 1 & 6,67 \\ \text { ACV } & 4 & 7,55 & 1 & 6,67 \\ \text { EVP } & 10 & 18,87 & 1 & 6,67 \\ \text { HU } & 6 & 11,32 & 1 & 6,67 \\ \text { OP } & 10 & 18,87 & 1 & 6,67 \\ \text { MCALB } & 0 & 0 & 0 & 0 \\ \text { DG } & 0 & 0 & 0 & 0 \\ \text { OTROS } & 1 & 1,89 & 1 & 6,67 \\ \text { TOTAL } & 53 & 32,92 & 15 & 28,30\end{array}$

Nota: DM2 = Diabetes Mellitus 2, HTA = Hipertensión arterial. IC = Insuficiencia Coronaria, ACV = Accidente cerebro vascular, EVP: enfermedad vascular Periférico, $\mathrm{HU}=$ Hiperuricemia, $\mathrm{OP}=$ ovario Poliquístico, $\mathrm{MCALB}=$ microalbuminuría, DG = Diabetes Gestacional.

\section{ANEXO 5.}

Tabla 3. Antecedentes Patológicos Familiares

$\begin{array}{lllll}\text { ENFERMEDAD } & \text { APF1 } & \% & \text { APF2 } & \% \\ \text { DM2 } & 58 & 36,02 & 27 & 16,77 \\ \text { OBSD } & 16 & 9,94 & 6 & 3,73 \\ \text { ACV } & 13 & 8,07 & 5 & 3,11 \\ \text { EC } & 21 & 13,04 & 5 & 3,11 \\ \text { HTA } & 58 & 36,02 & 8 & 4,97\end{array}$




$\begin{array}{lllll}\text { OTROS } & 24 & 14,91 & 1 & 0,62 \\ \text { TOTAL } & 190 & 118,1 & 52 & 32,30\end{array}$

Nota: $\mathrm{DM} 2=$ Diabetes Mellitus 2; OBSD = obesidad; $\mathrm{ACV}=$ Accidente Cerebro Vascular; EC = Enfermedad Coronaria; HTA = Hipertensión Arterial. 